\title{
Generating Pseudorandom Numbers From Various Distributions Using $\mathbf{C}++$
}

\author{
by Robert J. Yager
}

ARL-TN-613

June 2014

Approved for public release; distribution is unlimited. 


\section{NOTICES}

\section{Disclaimers}

The findings in this report are not to be construed as an official Department of the Army position unless so designated by other authorized documents.

Citation of manufacturer's or trade names does not constitute an official endorsement or approval of the use thereof.

Destroy this report when it is no longer needed. Do not return it to the originator. 


\title{
Army Research Laboratory
}

Aberdeen Proving Ground, MD 21005-5066

ARL-TN-613

June 2014

\section{Generating Pseudorandom Numbers From Various Distributions Using C++}

\author{
by Robert J. Yager
}

Weapons and Materials Research Directorate, ARL 


\section{REPORT DOCUMENTATION PAGE}

Public reporting burden for this collection of information is estimated to average 1 hour per response, including the time for reviewing instructions, searching existing data sources, gathering and maintaining the data needed, and completing and reviewing the collection information. Send comments regarding this burden estimate or any other aspect of this collection of information, including suggestions for reducing the burden, to Department of Defense, Washington Headquarters Services, Directorate for Information Operations and Reports (0704-0188), 1215 Jefferson Davis Highway, Suite 1204, Arlington, VA 22202-4302. Respondents should be aware that notwithstanding any other provision of law, no person shall be subject to any penalty for failing to comply with a collection of information if it does not display a currently valid OMB control number.

PLEASE DO NOT RETURN YOUR FORM TO THE ABOVE ADDRESS.

\begin{tabular}{|l|l|l}
\hline 1. REPORT DATE $(D D-M M-Y Y Y Y)$ & 2. REPORT TYPE & 3. DATES COVERED (From - To) \\
\hline
\end{tabular}

\begin{tabular}{l|l} 
June 2014 & Final \\
\hline 4. TITLE AND SUBTITLEE
\end{tabular}

4. TITLE AND SUBTITLE

October 2012-January 2014

Generating Pseudorandom Numbers From Various Distributions Using C++ 5a. CONTRACT NUMBER

\begin{tabular}{|c|c|}
\hline & 5b. GRANT NUMBER \\
\hline \multirow{2}{*}{$\begin{array}{l}\text { 6. AUTHOR(S) } \\
\text { Robert J. Yager }\end{array}$} & \\
\hline & 5e. TASK NUMBER \\
\hline $\begin{array}{l}\text { 7. PERFORMING ORGANIZATION NAME(S) AND ADDRESS(ES) } \\
\text { U.S. Army Research Laboratory } \\
\text { ATTN: RDRL-WML-A } \\
\text { Aberdeen Proving Ground, MD 21005-5066 }\end{array}$ & $\begin{array}{l}\text { 8. PERFORMING ORGANIZATION } \\
\text { REPORT NUMBER } \\
\text { ARL-TN-613 }\end{array}$ \\
\hline
\end{tabular}

Approved for public release; distribution is unlimited.

\section{SUPPLEMENTARY NOTES}

\section{ABSTRACT}

This report documents a set of functions, written in $\mathrm{C}++$, that can be used to generate pseudorandom numbers that have either uniform or normal distributions and pseudorandom integers that have either uniform or Poisson distributions. An implementation of the Mersenne twister algorithm, developed by Matsumoto and Nishimura, is included. The output from the Mersenne twister is used to generate the various distributions through the use of assorted transformation algorithms.

\section{SUBJECT TERMS}

pseudorandom, algorithm, Mersenne twister, $\mathrm{C}++$, normal, uniform, Poisson

\begin{tabular}{|l|l|l|l|l|l|}
\hline \multicolumn{2}{|l|}{ 16. SECURITY CLASSIFICATION OF: } & $\begin{array}{l}\text { 17. LIMITATION } \\
\text { OF ABSTRACT }\end{array}$ & $\begin{array}{l}\text { 18. NUMBER } \\
\text { OF PAGES }\end{array}$ & $\begin{array}{l}\text { 19a. NAME OF RESPONSIBLE PERSON } \\
\text { Robert J. Yager }\end{array}$ \\
\cline { 1 - 2 } $\begin{array}{l}\text { a. REPORT } \\
\text { Unclassified }\end{array}$ & $\begin{array}{l}\text { b. ABSTRACT } \\
\text { Unclassified }\end{array}$ & $\begin{array}{l}\text { c. THIS PAGE } \\
\text { Unclassified }\end{array}$ & UU & 28 & $\begin{array}{l}\text { 19b. TELEPHONE NUMBER (Include area code) } \\
410-278-6689\end{array}$ \\
\hline
\end{tabular}




\section{Contents}

$\begin{array}{ll}\text { Acknowledgments } & \text { v }\end{array}$

$\begin{array}{lr}\text { 1. Introduction } & 1\end{array}$

2. Generating Pseudorandom Integers Using the Mersenne Twister 19937 Algorithm The Rand() Function $\quad 1$

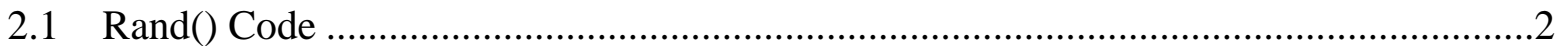

2.2 Rand() Template Classes ..................................................................................2

2.3 Rand() Parameters ........................................................................................2

2.4 Rand() Return Value .......................................................................................2

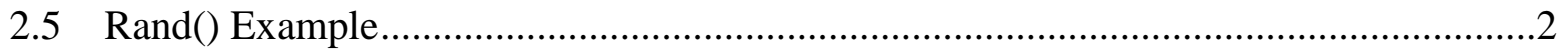

2.6 Comparison of the Rand() Function to Other Mersenne Twister Implementations........3

3. Initializing the Mersenne Twister - The Initialize() Function 4

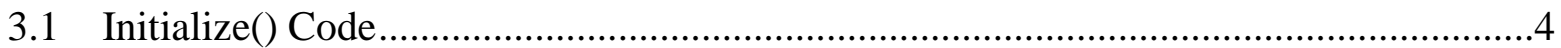

Initialize() Template Classes ...............................................................................4

Initialize() Parameters ..........................................................................................

4. Generating Uniformly Distributed Pseudorandom Numbers - The RandU() Function 5

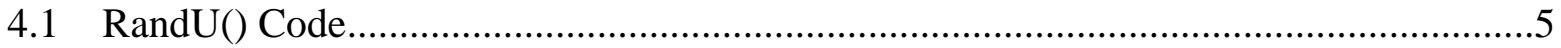

4.2 RandU() Template Classes ..................................................................................6

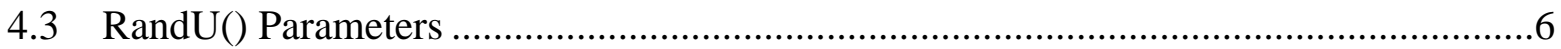

4.4 RandU() Return Value...................................................................................6

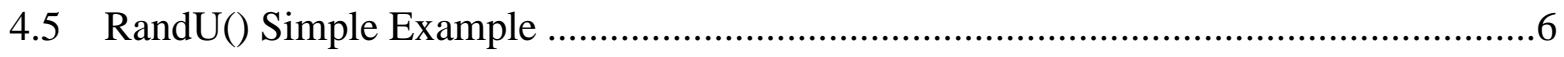

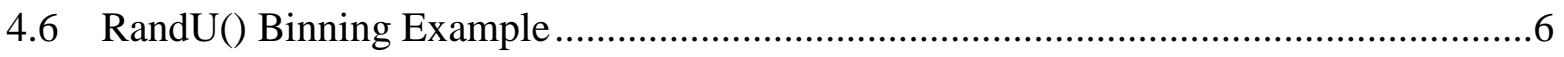

5. Generating Normally Distributed Pseudorandom Numbers - The RandN() Function 8

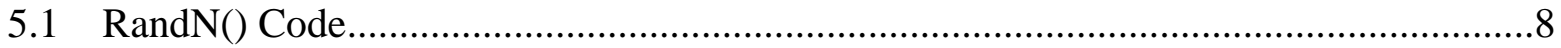

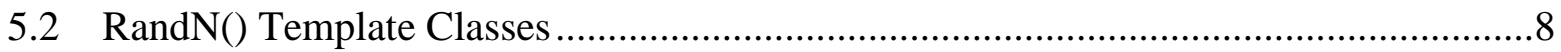

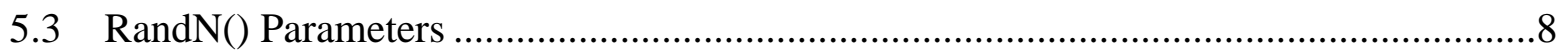

5.4 RandN() Return Value.................................................................................. 9 


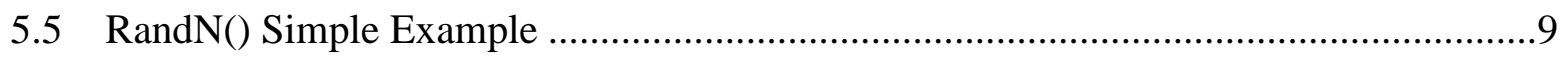

5.6 RandN() Binning Example ........................................................................ 9

6. Generating Uniformly Distributed Pseudorandom Integers - The RandI() Function 11



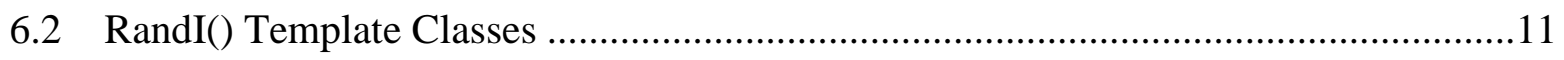

6.3 RandI() Parameters ........................................................................................... 11

6.4 RandI() Return Value .................................................................................11



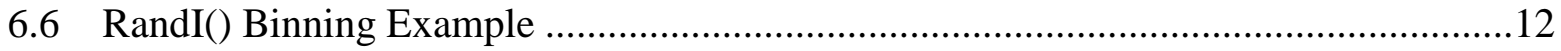

7. Generating Poisson-Distributed Pseudorandom Integers - The RandP() Function 13



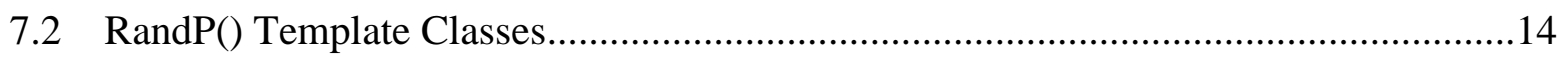

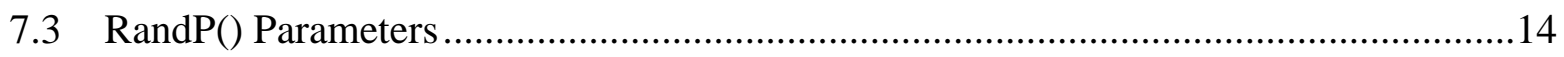

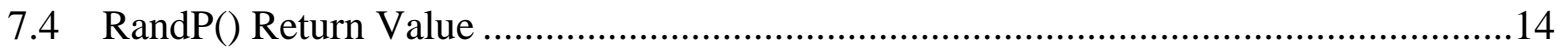

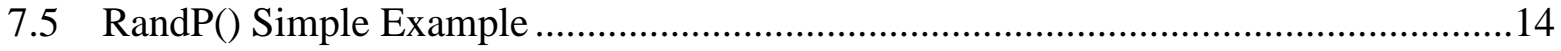

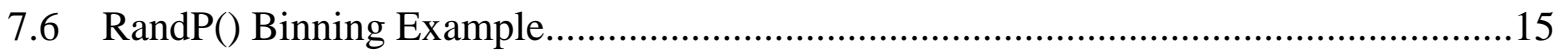

$\begin{array}{ll}\text { 8. Code Summary } & 16\end{array}$

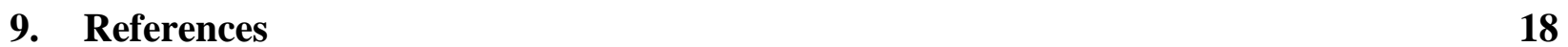

$\begin{array}{lr}\text { Distribution List } & 19\end{array}$ 


\section{Acknowledgments}

I would like to thank Mr. Benjamin Flanders and Dr. Benjamin Breech of the U.S. Army Research Laboratory's Weapons and Materials Research Directorate. Mr. Flanders and Dr. Breech provided technical and editorial recommendations that improved the quality of this report. 
INTENTIONALLY LEFT BLANK. 


\section{Introduction}

This report documents a set of functions, written in $\mathrm{C}++$, that can be used to generate pseudorandom numbers that have either uniform or normal distributions and pseudorandom integers that have either uniform or Poisson distributions. An implementation of the Mersenne twister algorithm, developed by Matsumoto and Nishimura (1), is included. The output from the Mersenne twister is used to generate the various distributions through the use of assorted transformation algorithms.

The functions presented here are offered as compiler-independent alternatives to functions defined by the new $\mathrm{C}++11$ standard (2). Although the new standard defines functions for generating pseudorandom numbers with uniform distributions, normal distributions, Poisson distributions, etc., it does not specify which algorithms will be used to generate those pseudorandom numbers. Thus, compilers that conform to the new standard will all generate pseudorandom numbers from the various distributions, but the actual numbers that are generated may differ from compiler to compiler.

The functions presented in this report have been grouped into the yRandom namespace, which is summarized at the end of this report.

\section{Generating Pseudorandom Integers Using the Mersenne Twister 19937 Algorithm - The Rand() Function}

The Rand() function uses the Mersenne twister 19937 algorithm to generate uniformly distributed pseudorandom integers in the interval $\left[0,2^{32}\right)$. According to Matsumoto and Nishimura, the algorithm has a period of $2^{19937}-1$ and passes the Diehard tests for statistical randomness.

The state of the Mersenne twister is stored in an array of 625 32-bit unsigned integers, which is passed as an argument to the Rand() function. The initial state of the Mersenne twister can be set using the Initialize() function (see section 3). The Initialize() function uses a user-supplied 32-bit integer to seed a simple pseudorandom number generator that generates the initial state integers. This effectively allows for the creation of multiple pseudorandom number generators (as many as $2^{32}$ ) that can each produce independent, reproducible sequences of pseudorandom integers. 
The code contained in the Rand() function differs from the code that is presented by Matsumoto and Nishimura. To avoid using the modulo operator, their code calculates all of the algorithm's 624 unsigned state integers once out of every 624 function calls. In contrast, the Rand() function uses the ternary operator to avoid using the modulo operator.

\subsection{Rand() Code}

template $<$ class $T>T$ Rand $(/ /<=====================$ MERSENNE TWISTER (19937) PRNG

T I $[625])\{/ /<-S T A T E$ OF MERSENNE TWISTER (FOR T, USE A 32-BIT UNSIGNED INT)

$T \quad i=I[624], j=i<623$ ? $i+1: 0, y=I[i] \& 0 \times 80000000 \mid I[j] \& 0 \times 7 f f f f f f f ;$

$y=I[i]=I[i<227 \text { ? } i+397: i-227]^{\wedge} y>>1^{\wedge}(y \& 1) * 0 x 9908 b \theta d f, I[624]=j$;

return $y^{\wedge}\left(y^{\wedge}=\left(y^{\wedge}=\left(y^{\wedge}=y>>11\right)<<780 x 9 d 2 c 5680\right)<<15 \& 0 x e f c 60000\right)>>18$;

\}// YAGENAUT@GMAIL.COM LAST UPDDATED 21JJAN2014

\subsection{Rand() Template Classes}

T T should be a 32-bit unsigned integer type. Greater than 32-bit unsigned integer types can be used, but performance may suffer.

\subsection{Rand() Parameters}

I I points to a 625-element array that contains the state of the Mersenne twister algorithm. Each time the Rand() function is called, the array pointed to by $\mathbf{I}$ is modified. The initial state of the array should be set using the Initialize() function (see section 3).

\subsection{Rand() Return Value}

The Rand() function returns uniformly distributed pseudorandom integers in the interval $\left[0,2^{32}\right.$ ).

\subsection{Rand() Example}

The following example uses the Rand() function to generate one billion pseudorandom integers. The Initialize() function, introduced in section 3, is used to set the initial state of the Mersenne twister.

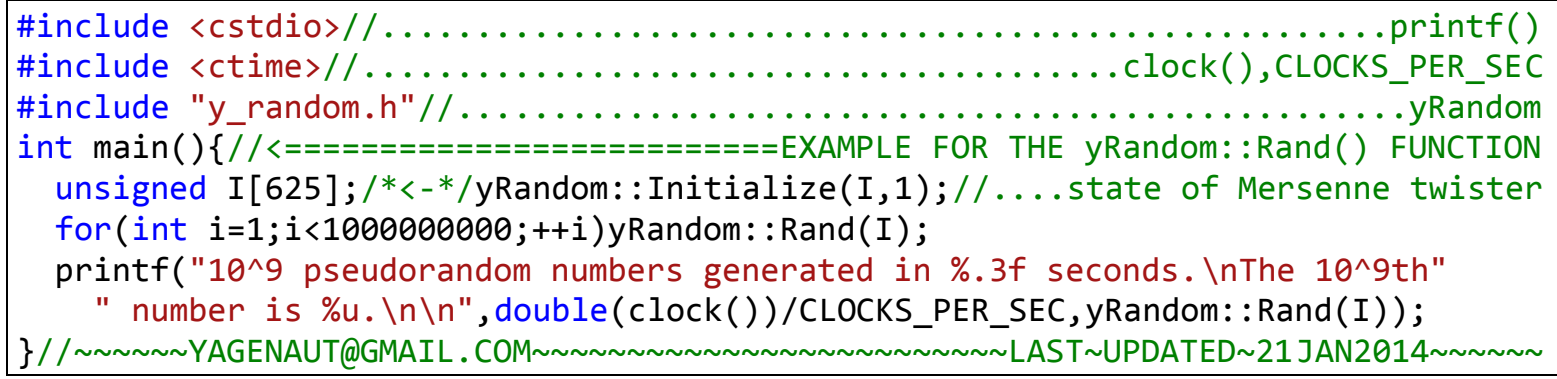




\section{OUTPUT:}

$10^{\wedge} 9$ pseudorandom numbers generated in 1.778 seconds.

The $10^{\wedge}$ 9th number is 2716480233 .

\subsection{Comparison of the Rand() Function to Other Mersenne Twister Implementations}

The following example compares the Rand() function to Matsumoto and Nishimura's genrand() function, as well as to the $\mathrm{C}++11$ built-in Mersenne twister implementation. The example code demonstrates that the three implementations produce identical output (at least for the first $10^{9}$ values and with a seed value of 1). Note that time values will vary based on computer specifications, compiler, compiler settings, etc.

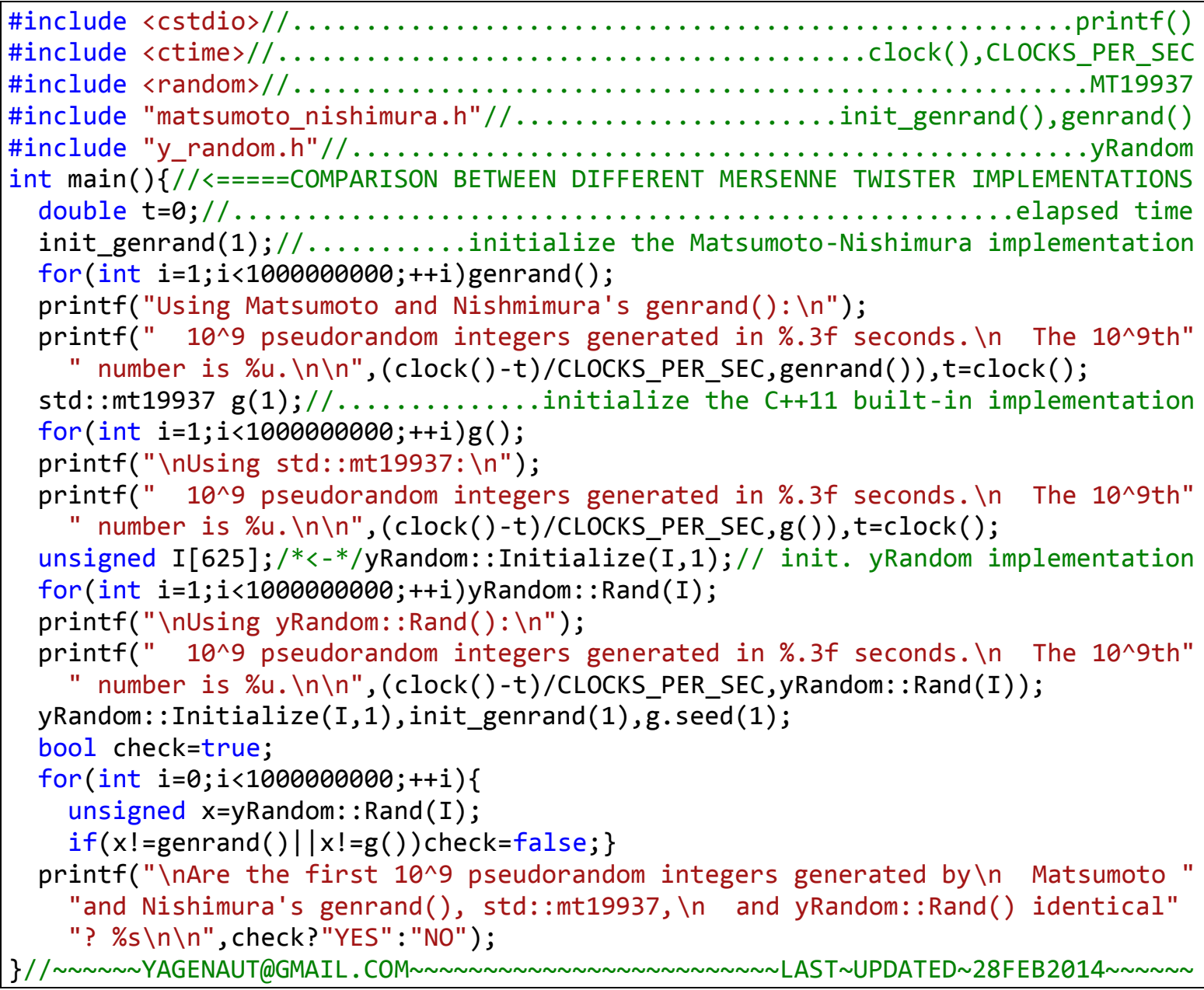




\section{OUTPUT:}

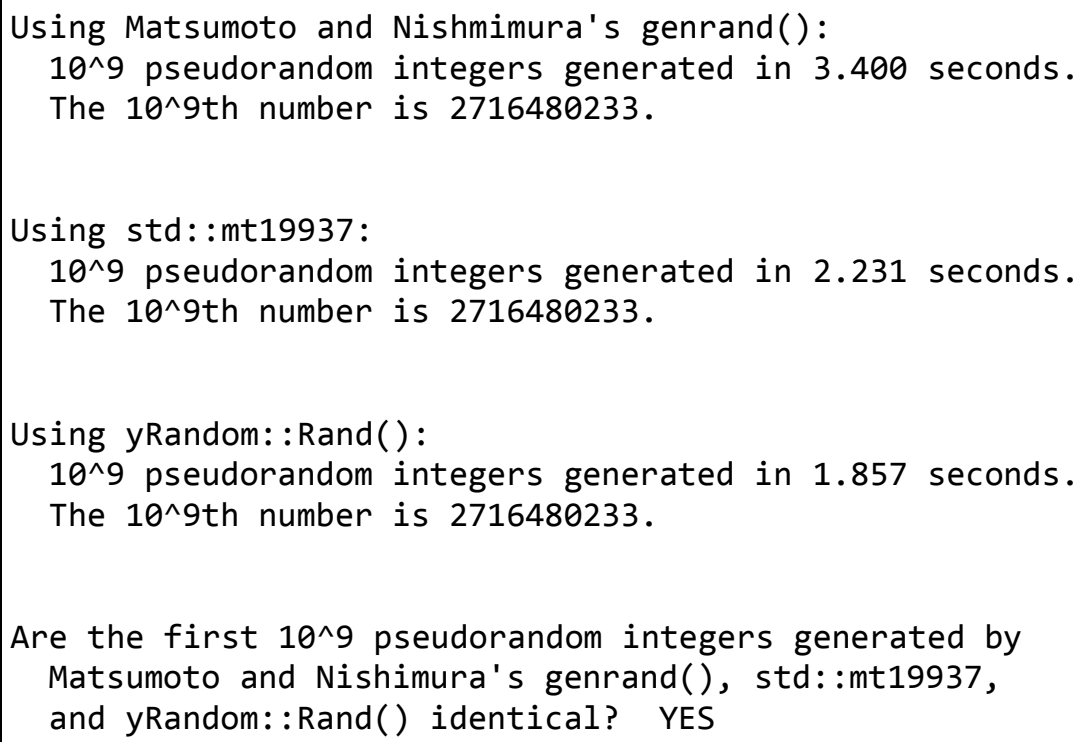

\section{Initializing the Mersenne Twister - The Initialize() Function}

The Initialize() function uses an algorithm presented by Nishimura and Matsumoto (3) to initialize the 625-element array that is used to store the state of the Mersenne twister algorithm.

\subsection{Initialize() Code}

template<class T>void Initialize(//<======INITIALIZE STATE OF MERSENNE TWISTER T I [625],//<--STATE OF MERSENNE TWISTER (FOR T, USE A 32-BIT UNSIGNED INT)

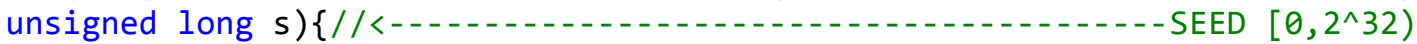
I $[0]=$ s\&0xffffffff, $I[624]=0$;

for (int $i=1 ; i<624 ;++i) I[i]=\left(1812433253^{*}\left(I[i-1]^{\wedge} I[i-1]>>30\right)+i\right) \& 0 x f f f f f f f f$; \}// YAGENAUT@GMAIL.COM LAST UPDATED 21JAN2014

\subsection{Initialize() Template Classes}

T T should be a 32-bit unsigned integer type. Greater than 32-bit types can be used, but performance may suffer. 


\subsection{Initialize() Parameters}

I I points to storage for a 625 -element array that is used to store the state of the Mersenne twister algorithm.

$\mathbf{S}$ $\mathbf{s}$ specifies the seed for the algorithm that is used to generate the initial state of the Mersenne twister algorithm. $\mathbf{s}$ can be any value in the interval $\left[0,2^{32}\right)$.

\section{Generating Uniformly Distributed Pseudorandom Numbers - The RandU() Function}

The RandU() function can be used to generate uniformly distributed pseudorandom numbers that conform to the probability density function given by equation 1 .

$$
f(x)=\left\{\begin{array}{cl}
\frac{1}{b-a}, & \text { for } a<x \leq b \\
0, & \text { otherwise }
\end{array}\right.
$$

Note that the $\mathrm{C}++$ standards document defines a slightly different probability density function for uniformly distributed pseudorandom numbers $(a \leq x<b$ rather than $a<x \leq b)$. The decision to exclude the lower bound from the distribution rather than the upper bound was based on the belief that it would be more likely to help the user to avoid a divide-by-zero error.

The RandU() function uses the transformation presented in equation 2, where $r$ is a uniformly distributed pseudorandom number in the interval $(0,1]$.

$$
x=a+(b-a) r
$$

Equation 3 can be used to generate $r$ given $q$, a uniformly distributed pseudorandom integer in the interval $\left[0,2^{32}\right)$.

$$
r=\frac{q+1}{2^{32}}
$$

\subsection{RandU() Code}

template $<$ class T>double RandU(//<====UNIFORMLY DISTRIBUTED PSEUDORANDOM DOUBLE

T I[625],//<--STATE OF MERSENNE TWISTER (FOR T, USE A 32-BIT UNSIGNED INT)

double a, double b) $\{/ /<-\cdots-\ldots-1,-$ LOWER \& UPPER BOUNDARIES OF DISTRIBUTION

return $a+(b-a) *(\operatorname{Rand}(I)+1) / .4294967296 ; / / \ldots \ldots \ldots \ldots$ for $a=0$ and $b=1,(0,1]$

\}// YAGENAUT@GMAIL.COM LAST UPDATED 21JAN2014 


\subsection{RandU() Template Classes}

T T should be a 32-bit unsigned integer type. Greater than 32-bit types can be used, but performance may suffer.

\subsection{RandU() Parameters}

I I points to an array that contains the state of the Mersenne twister algorithm.

a a specifies $a$, the lower bound for the distribution.

b b specifies $b$, the upper bound for the distribution.

\subsection{RandU() Return Value}

The RandU() function returns uniformly distributed pseudorandom numbers in the interval (a,b]. Note that, due to limitations inherent in the storage of double precision numbers, the return value is not guaranteed to be distinct from $\mathbf{a}$ in all cases (such as when $|\mathbf{a}|>>|\mathbf{a}-\mathbf{b}|$ ).

\subsection{RandU() Simple Example}

The following example uses the RandU() function to generate and sum one billion uniformly distributed pseudorandom numbers in the interval $(2,6]$.

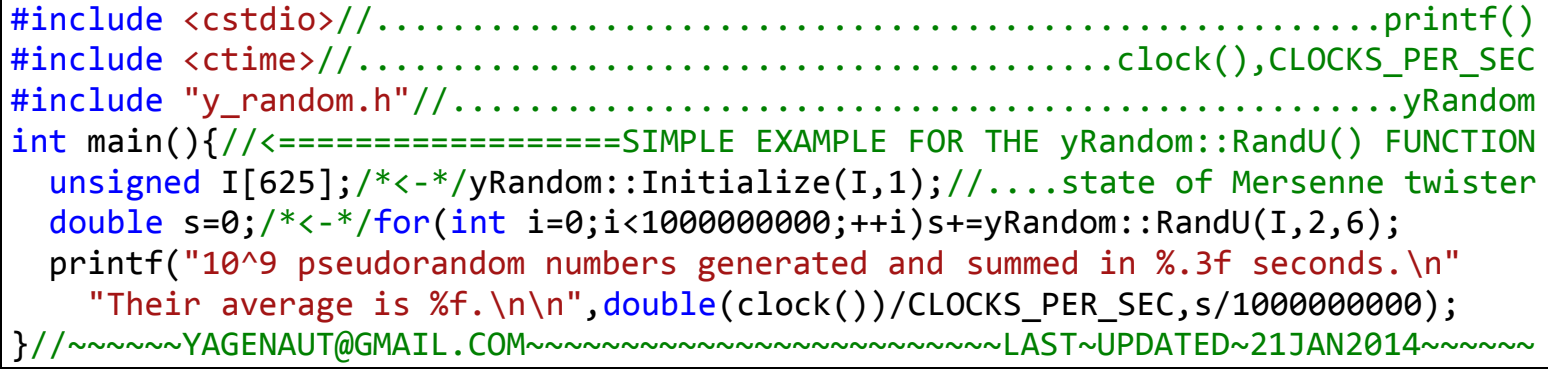

\section{OUTPUT:}

$10^{\wedge} 9$ pseudorandom numbers generated and summed in 8.255 seconds.

Their average is 3.999977 .

\subsection{RandU() Binning Example}

The following example uses the RandU() function to generate and bin one billion uniformly distributed pseudorandom numbers in the interval $(2,6]$. 


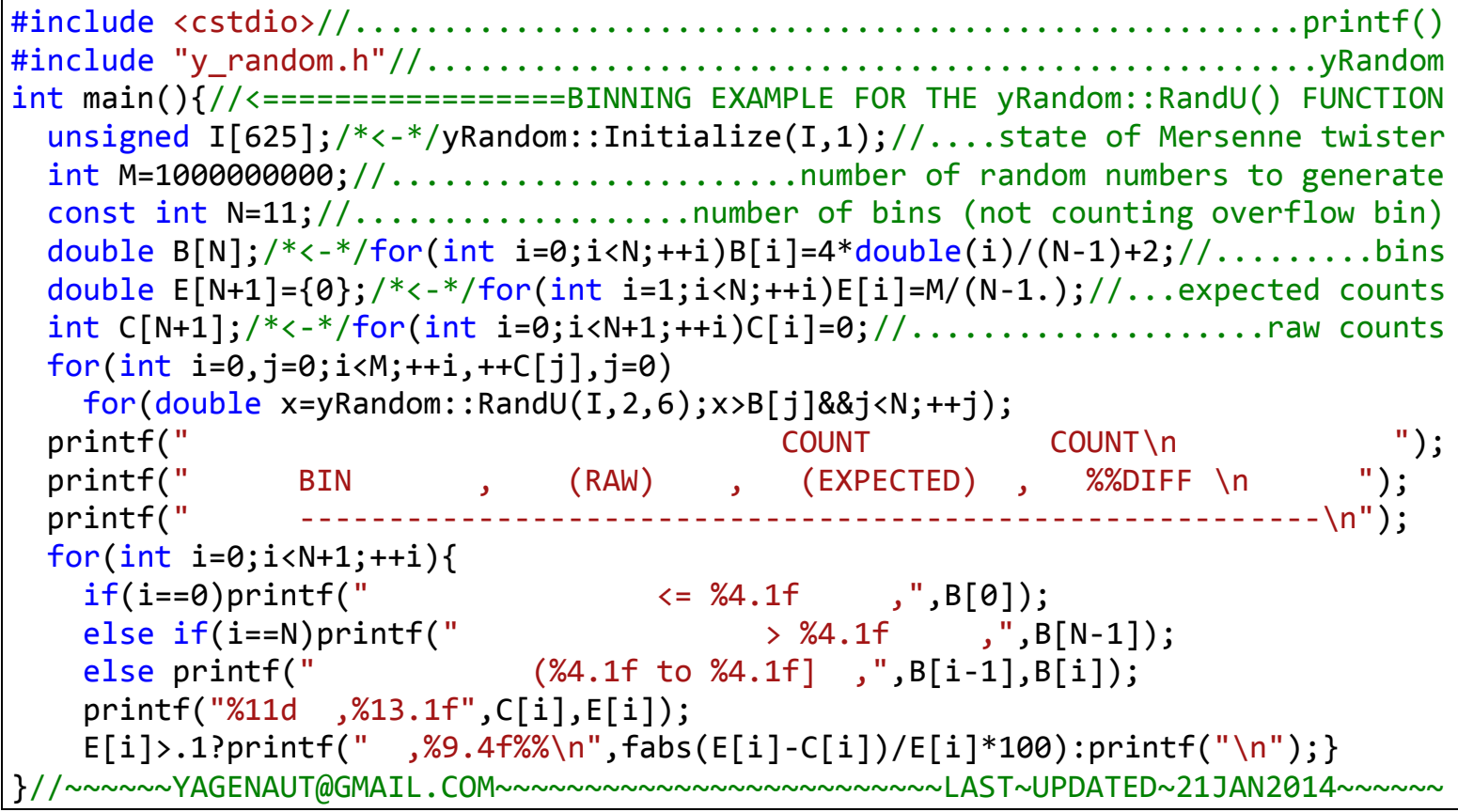

\section{OUTPUT:}

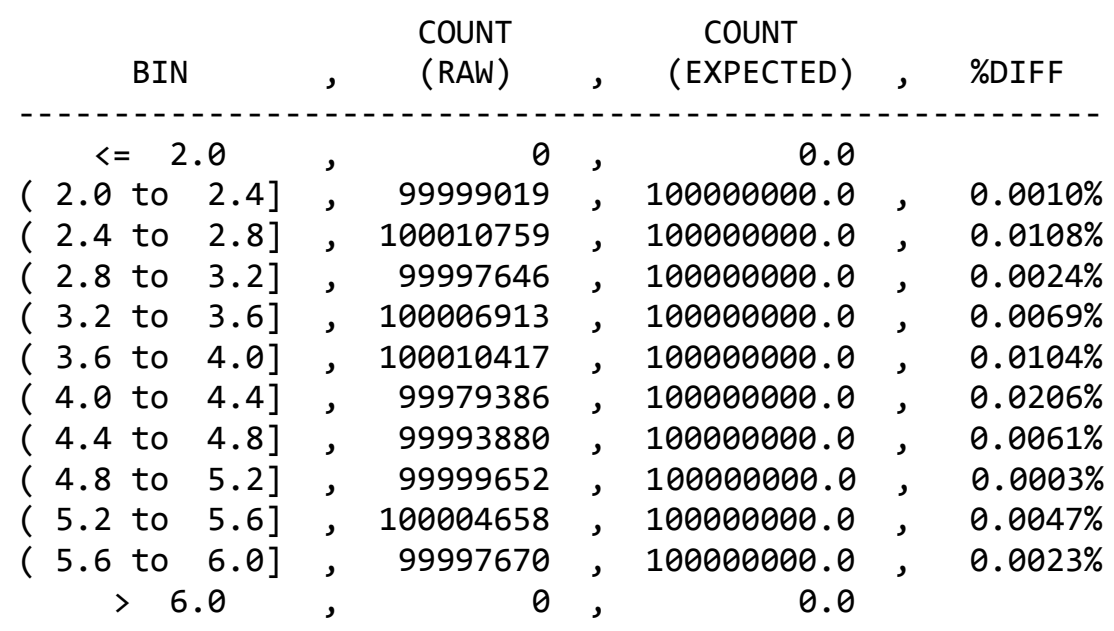




\section{Generating Normally Distributed Pseudorandom Numbers - The RandN() Function}

The RandN() function can be used to generate normally distributed pseudorandom numbers that conform to the probability density function given by equation 4 , where $\mu$ is the mean of the distribution and $\sigma$ is the standard deviation.

$$
f(x)=\frac{1}{\sigma \sqrt{2 \pi}} e^{-\frac{(x-\mu)^{2}}{2 \sigma^{2}}}
$$

The RandN() function uses the Box-Muller transform (4) to generate normally distributed pseudorandom numbers:

$$
x=\mu+\sigma \sqrt{-2 \ln \left(r_{a}\right)} \cos \left(2 \pi r_{b}\right)
$$

where $r_{a}$ and $r_{b}$ are uniformly distributed pseudorandom numbers in the interval $(0,1]$. Equation 3 can be used to generate $r_{a}$ and $r_{b}$ given $q_{a}$ and $q_{b}$, two uniformly distributed pseudorandom integers in the interval $\left[0,2^{32}\right)$.

\subsection{RandN() Code}

template $<$ class $T>$ double RandN $(/ /<=====$ NORMALLY DISTRIBUTED PSEUDORANDOM DOUBLE T I [625],//<--STATE OF MERSENNE TWISTER (FOR T, USE A 32-BIT UNSIGNED INT) double $m$, double $s)\{/ /<--.---M E A N \&$ STANDARD DEVIATION OF DISTRIBUTION return $\mathrm{m}+\mathrm{s}^{*} \operatorname{sqrt}(-2 * \log ((\operatorname{Rand}(\mathrm{I})+1) / 4294967296)$.

$* \cos (1.4629180792671596 \mathrm{E}-9 *(\operatorname{Rand}(\mathrm{I})+1)$.$) ;$

\}// YAGENAUT@GMAIL.COM LAST UPDDATED 21JAN2014

\subsection{RandN() Template Classes}

T T should be a 32-bit unsigned integer type. Greater than 32-bit types can be used, but performance may suffer.

\subsection{RandN() Parameters}

I I points to an array that contains the state of the Mersenne twister algorithm.

m $\quad$ m specifies $\mu$, the mean of the distribution.

$\mathbf{S}$ s specifies $\sigma$, the standard deviation of the distribution. 


\subsection{RandN() Return Value}

The RandN() function returns normally distributed pseudorandom numbers.

\subsection{RandN() Simple Example}

The following example uses the $\operatorname{RandN}()$ function to generate and sum one billion normally distributed pseudorandom numbers with $\mu=4$ and $\sigma=0.5$.



\section{OUTPUT:}

10^9 pseudorandom numbers generated and summed in 46.213 seconds.

Their average is 3.999998 .

\subsection{RandN() Binning Example}

The following example uses the RandN() function to generate and bin one billion normally distributed pseudorandom numbers with $\mu=4$ and $\sigma=0.5$. The Erf() function is an implementation of an algorithm presented by Abramowitz and Stegun (5). 


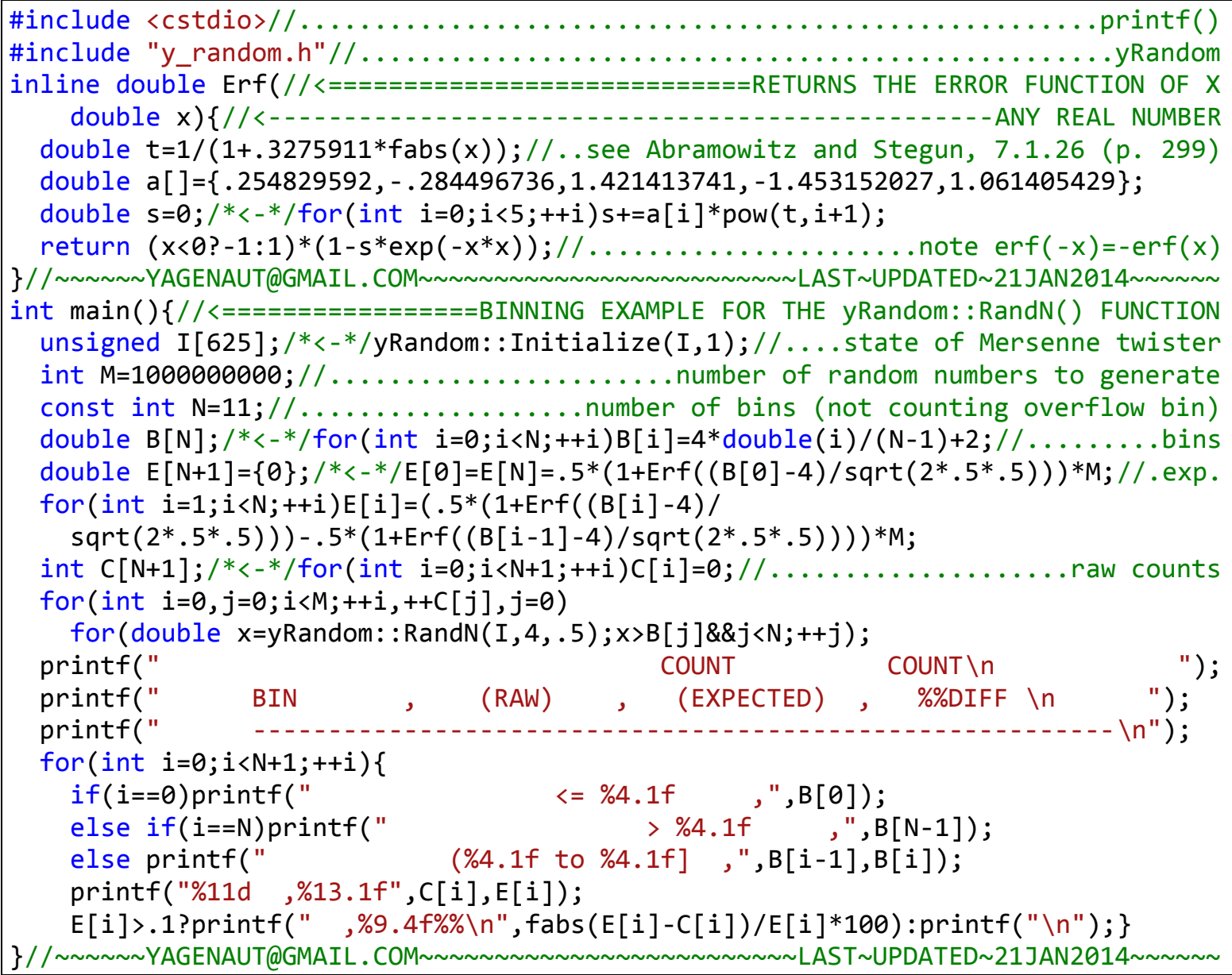

\section{OUTPUT:}

\begin{tabular}{|c|c|c|c|c|c|c|c|}
\hline \multicolumn{2}{|c|}{ BIN } & , & $\begin{array}{l}\text { COUNT } \\
\text { (RAW) }\end{array}$ & , & $\begin{array}{c}\text { COUNT } \\
(\text { EXPECTED) }\end{array}$ & , & \%DIFF \\
\hline \multicolumn{2}{|c|}{$<=2.0$} & , & 31839 & , & 31686.0 & & $0.4827 \%$ \\
\hline $2.0 \mathrm{t}$ & 2.4] & s & 655830 & & 655516.1 & & $0.0479 \%$ \\
\hline $2.4 t$ & $2.8]$ & & 7507620 & & 7510327.1 & & $0.0360 \%$ \\
\hline $2.8 \mathrm{t}$ & $3.2]$ & & 46597020 & & 46601762.1 & & $0.0102 \%$ \\
\hline $3.2 \mathrm{t}$ & $3.6]$ & & 157066112 & & 157056047.3 & & $0.0064 \%$ \\
\hline $3.6 \mathrm{t}$ & $4.0]$ & & 288150018 & & 288144661.9 & & $0.0019 \%$ \\
\hline $4.0 \mathrm{t}$ & 4.4] & & 288145262 & & 288144660.9 & , & $0.0002 \%$ \\
\hline $4.4 t$ & $4.8]$ & & 157043835 & & 157056047.3 & & $0.0078 \%$ \\
\hline $4.8 t$ & $5.2]$ & & 46607503 & & 46601762.1 & & $0.0123 \%$ \\
\hline $5.2 \mathrm{t}$ & $5.6]$ & & 7508108 & & 7510327.1 & & $0.0295 \%$ \\
\hline 5.6 & $6.0]$ & & 655295 & & 655516.1 & & $0.0337 \%$ \\
\hline$>$ & & & 31558 & & 31686.0 & & $0.4041 \%$ \\
\hline
\end{tabular}




\section{Generating Uniformly Distributed Pseudorandom Integers - The RandI() Function}

The RandI() function can be used to generate uniformly distributed pseudorandom integers that conform to the probability density function given by equation 6 .

$$
f(k)=\left\{\begin{array}{cc}
\frac{1}{b-a+1}, & \text { for } a \leq k \leq b \\
0, & \text { otherwise }
\end{array}\right.
$$

The RandI() function uses the transformation presented in equation 7 , where $q$ is a uniformly distributed pseudorandom integer in the interval $\left[0,2^{32}\right)$.

$$
k=a+\operatorname{trunc}\left(\frac{q}{2^{32}}(b-a+1)\right)
$$

\subsection{RandI() Code}

template<class T>long RandI(//<=========UNIFORMLY DISTRIBUTED PSEUDORANDOM INT

T I [625],//<--STATE OF MERSENNE TWISTER (FOR T, USE A 32 -BIT UNSIGNED INT)

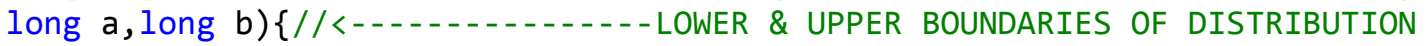

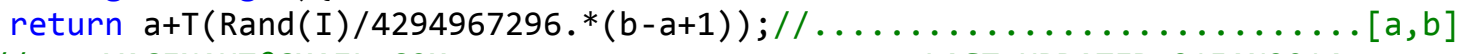

\}// YAGENAUT@GMAIL.COM LAST UPDATED 21JAN2014

\subsection{RandI() Template Classes}

T T should be a 32-bit unsigned integer type. Greater than 32-bit types can be used, but performance may suffer.

\subsection{RandI() Parameters}

I I points to an array that contains the state of the Mersenne twister algorithm.

a a specifies $a$, the lower bound for the distribution.

b b specifies $b$, the upper bound for the distribution.

\subsection{RandI() Return Value}

The RandI() function returns a uniformly distributed pseudorandom integer in the interval [a,b].

\subsection{RandI() Simple Example}

The following example uses the RandI() function to generate and sum one billion uniformly distributed pseudorandom integers in the interval $[-5,4]$. 


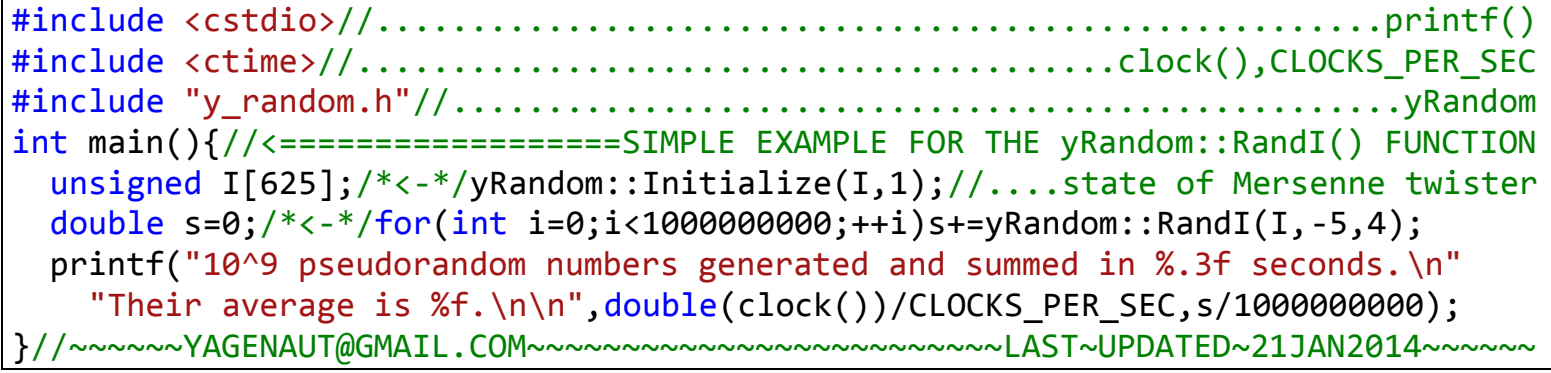

\section{OUTPUT:}

$10^{\wedge} 9$ pseudorandom numbers generated and summed in 10.140 seconds.

Their average is -0.500057 .

\subsection{RandI() Binning Example}

The following example uses the RandI() function to generate and bin one billion uniformly distributed pseudorandom integers in the interval $[-5,4]$.

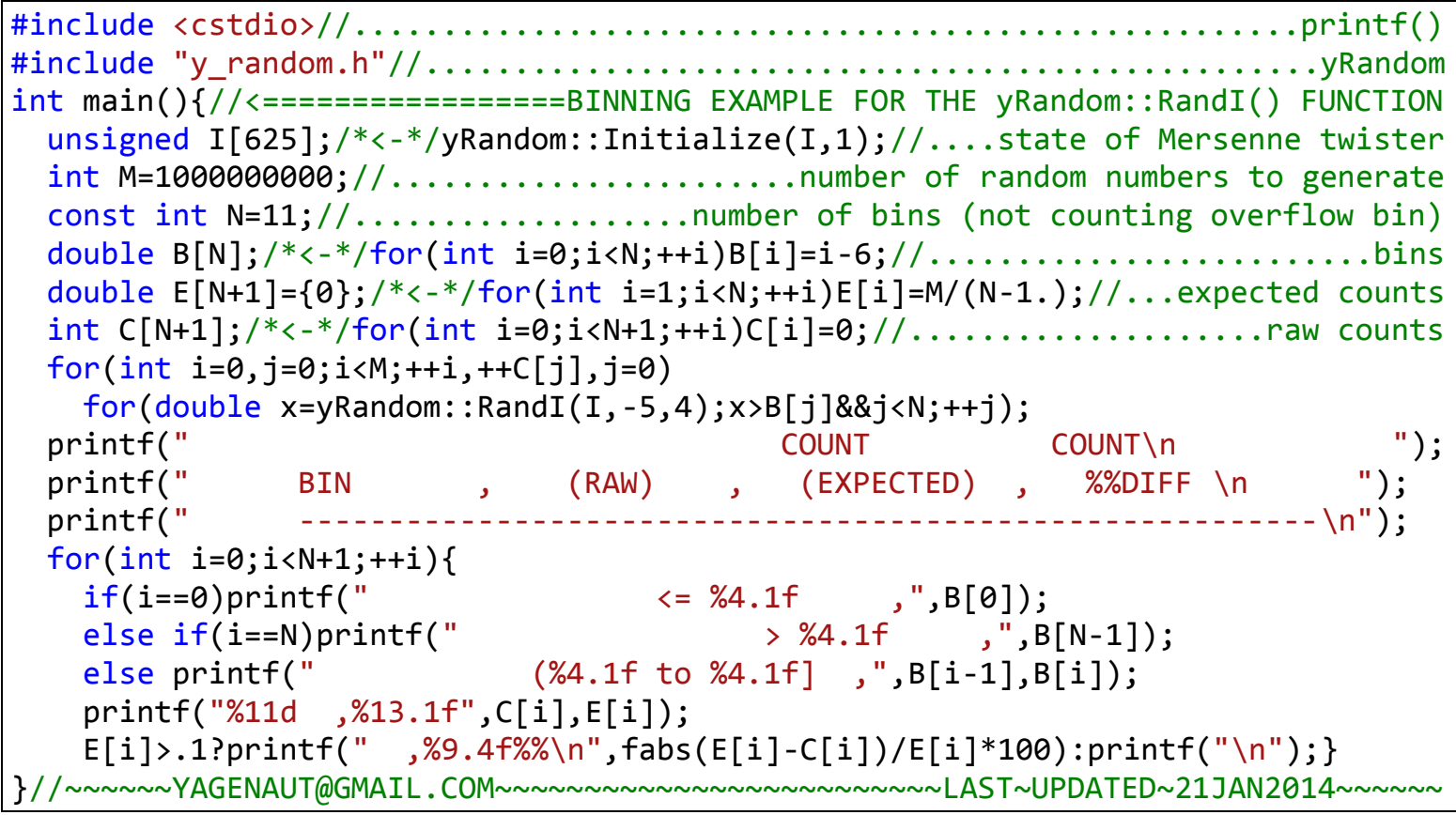




\section{OUTPUT:}

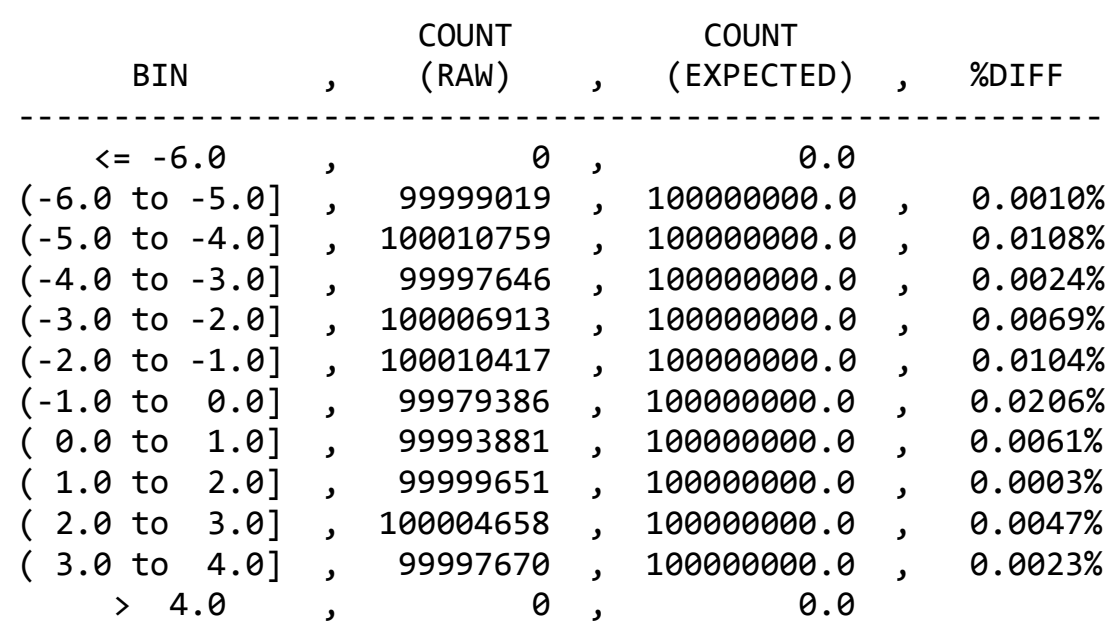

\section{Generating Poisson-Distributed Pseudorandom Integers - The RandP() Function}

The RandP() function can be used to generate Poisson distributed pseudorandom integers that conform to the probability density function given by equation 8 , where $\mu$ is the mean of the distribution.

$$
f(k)=\frac{\mu^{k} e^{-\mu}}{k !}
$$

The $\operatorname{RandP}()$ function uses a transformation described by Knuth (6) to generate Poissondistributed pseudorandom integers by finding the largest $k$ that satisfies equation 9 .

$$
\prod_{i=0}^{k} r_{i}>e^{-\mu}
$$

where $r_{i}$ is a uniformly distributed pseudorandom number in the interval $(0,1]$. Equation 3 can be used to generate each $r_{i}$ given $q_{i}$, a uniformly distributed pseudorandom integer in the interval $\left[0,2^{32}\right)$.

Note that the for large $\mu$, the $\operatorname{RandP}()$ function is slow. This problem can be overcome by making use of the fact that, for large $\mu$, Poisson distributions are approximately normal. 


\subsection{RandP() Code}

template<class $T>T$ RandP(//<=====POISSON-DISTRIBUTED PSEUDORANDOM UNSIGNED INT

T I [625],//<--STATE OF MERSENNE TWISTER (FOR T, USE A 32 -BIT UNSIGNED INT)

double $m)\{/ /<\ldots-\ldots-\ldots$ MEAN (MUST BE GREATER THAN ZERO)

$\mathrm{T} \mathrm{k}=0$; /*<-*/for (double $\left.\mathrm{P}=1, \mathrm{E}=\exp (-\mathrm{m}) ; \mathrm{P}>\mathrm{E} ; \mathrm{P}^{*}=(\operatorname{Rand}(\mathrm{I})+1) / 4294967296.\right)++\mathrm{k}$;

return $\mathrm{k}-1$;

\} // YAGENAUT@GMAIL.COM LAST UPDATED 21JAN2014

\subsection{RandP() Template Classes}

T T should be a 32-bit unsigned integer type. Greater than 32-bit types can be used, but performance may suffer.

\subsection{RandP() Parameters}

I I points to an array that contains the state of the Mersenne twister algorithm.

m $\quad$ m specifies $\mu$, the mean of a Poisson distribution.

\subsection{RandP() Return Value}

The RandP() function returns a Poisson-distributed pseudorandom unsigned integer.

\subsection{RandP() Simple Example}

The following example uses the RandP() function to generate and sum one billion Poissondistributed pseudorandom integers with $\mu=1$.



\section{OUTPUT:}

$10^{\wedge} 9$ pseudorandom numbers generated and summed in 35.708 seconds. Their average is 1.000013 . 


\subsection{RandP() Binning Example}

The following example uses the RandP() function to generate and bin one billion Poisson distributed pseudorandom integers with $\mu=1$.

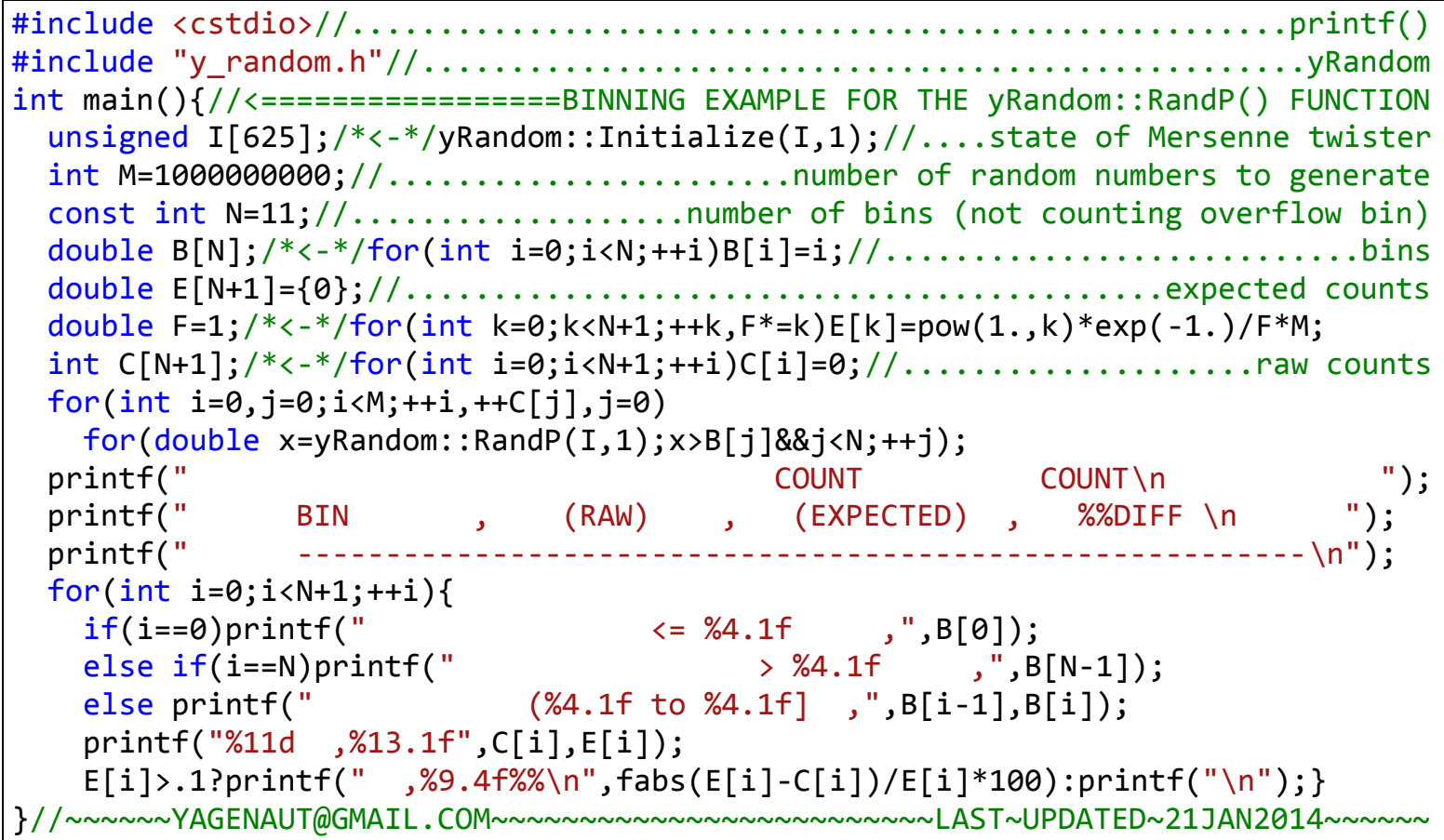

\section{OUTPUT:}

\begin{tabular}{|c|c|c|c|c|c|c|c|}
\hline \multicolumn{2}{|c|}{ BIN } & , & $\begin{array}{l}\text { COUNT } \\
\text { (RAW) }\end{array}$ & , & $\begin{array}{c}\text { COUNT } \\
(\text { EXPECTED) }\end{array}$ & & \%DIFF \\
\hline \multicolumn{2}{|c|}{$<=0.0$} & , & 367886090 & , & 367879441.2 & & $0.0018 \%$ \\
\hline$(0.0 t$ & $1.0]$ & , & 367871283 & , & 367879441.2 & , & $0.0022 \%$ \\
\hline$(1.0 t$ & $2.0]$ & & 183927644 & & 183939720.6 & , & $0.0066 \%$ \\
\hline$(2.0 t$ & $3.0]$ & & 61321837 & & 61313240.2 & , & $0.0140 \%$ \\
\hline $3.0 t$ & $4.0]$ & & 15332140 & & 15328310.0 & & $0.0250 \%$ \\
\hline $4.0 t$ & $5.0]$ & & 3067729 & & 3065662.0 & & $0.0674 \%$ \\
\hline $5.0 t$ & $6.0]$ & & 510230 & & 510943.7 & & $0.1397 \%$ \\
\hline $6.0 t$ & $7.0]$ & & 72871 & & 72992.0 & & $0.1657 \%$ \\
\hline $7.0 t$ & $8.0]$ & & 9053 & & 9124.0 & & $0.7781 \%$ \\
\hline 8.0 & $9.0]$ & & 999 & & 1013.8 & & $1.4576 \%$ \\
\hline $9.0 t$ & $10.0]$ & & 112 & & 101.4 & & $10.4779 \%$ \\
\hline & .0 & & 12 & & 9.2 & & $30.2061 \%$ \\
\hline
\end{tabular}




\section{Code Summary}

A summary sheet is provided at the end of this report. It presents the yRandom namespace, which contains the six functions that are described in this report. 


\section{yRandom Summary}

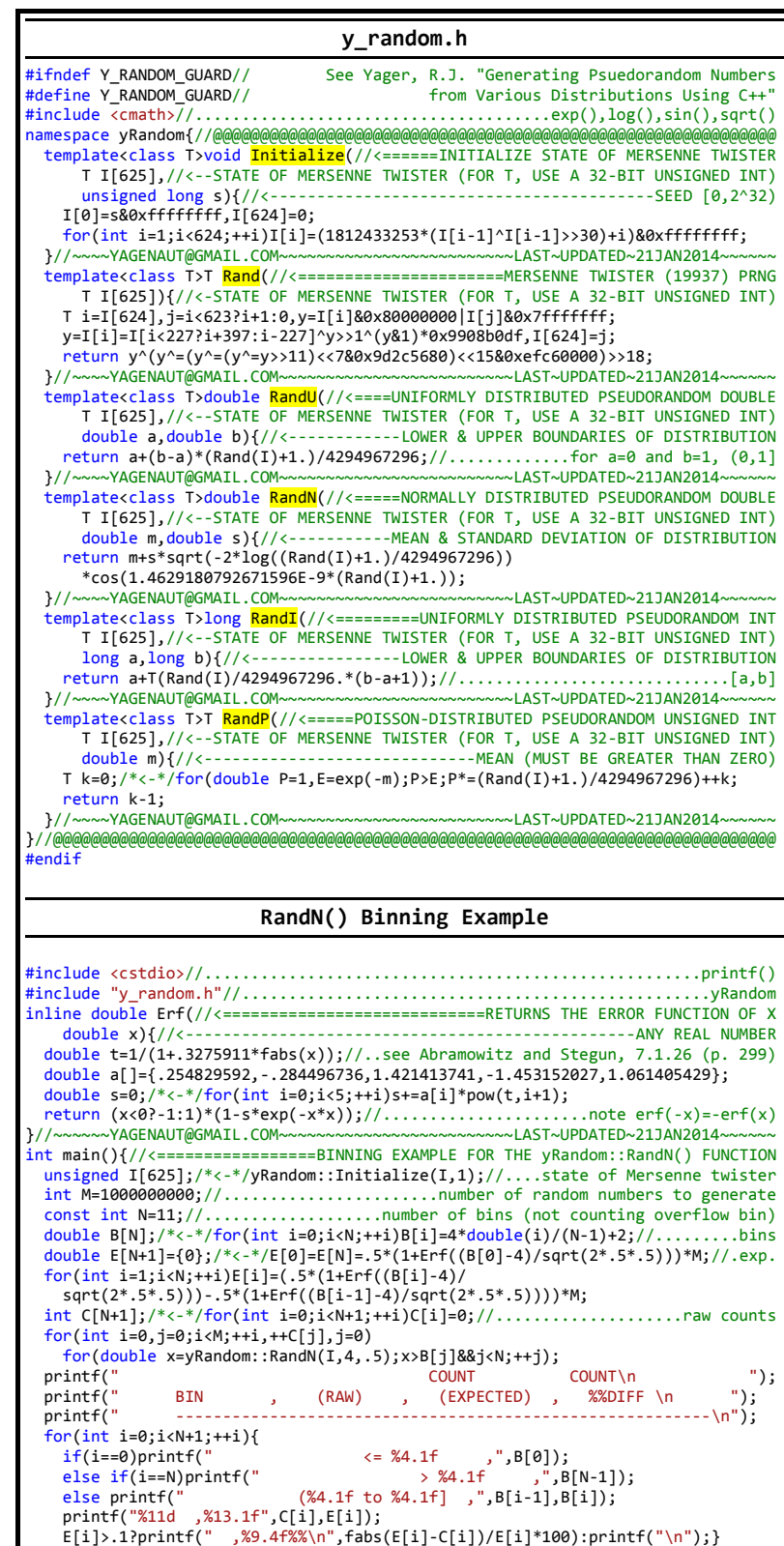

OUTPUT:

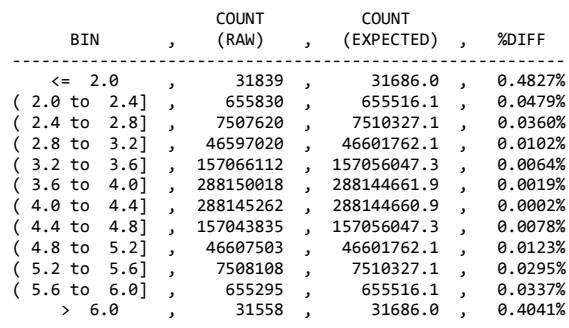

\begin{tabular}{|c|}
\hline Rand() Example \\
\hline 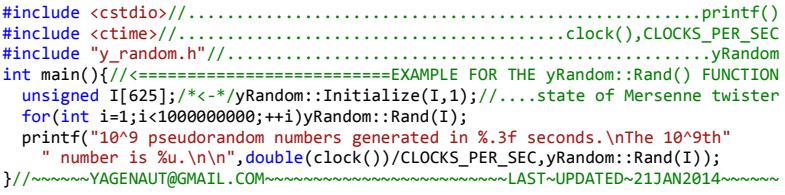 \\
\hline
\end{tabular}

OUTPUT:

$10^{\wedge} 9$ pseudorandom numbers generated in 1.778 seconds.

The $10^{\wedge} 9$ th number is 2716480233 .

RandU() Probability Density Function and Simple Example

$$
f(x)=\left\{\begin{array}{cl}
\frac{1}{b-a}, & \text { for } a<x \leq b \\
0, & \text { otherwise }
\end{array}\right.
$$



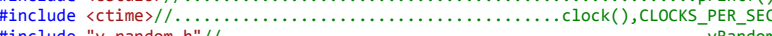

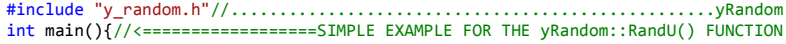

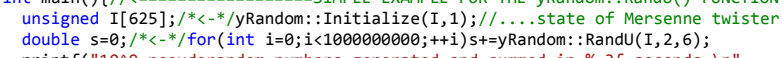
double $s=0 ; / *<-* /$ for (int $i=0 ; i<1000000000 ;++i) s+=y$ Random: : RandU $(I, 2,6)$;
printf(" $10^{\wedge} 9$ pseudorandom numbers generated and summed in $\% .3 f$ seconds. In"

"Their average is \%f. \n\n", double(clock())/CLOCKS_PER_SEC, s/10000000000);

\}// YAGENAUT@GMAIL.COM LAST UUPDATED 21JAN2014

OUTPUT:

$10^{\wedge} 9$ pseudorandom numbers generated and summed in 8.255 seconds.

Their average is 3.999977 .

RandN() Probability Density Function and Simple Example

$$
f(x)=\frac{1}{\sigma \sqrt{2 \pi}} e^{-\frac{(x-\mu)^{2}}{2 \sigma^{2}}}
$$

\#include $\langle$ cstdio $>/ /$

\#include <ctime $/ / \ldots$.
\#include $\mathrm{y}$ random. $\mathrm{h} " / /$

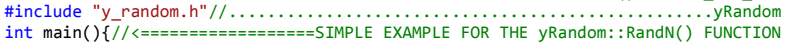

unsigned I[625];/*<-*/yRandom: : Initialize $(I, 1) ; / / \ldots$ state of Mersenne twiste

double $s=0 ; / *<-* /$ for (int $i=0 ; i<1000000000 ;++i) s+=y$ Random: : RandN $(I, 4, .5)$;

"Their average is \%f. \n\n", double(clock())/CLOCKS_PER_SEC, s/1000000000);

\}// "T YAGENAUT@GMAIL.COM a , d LAST UPDATED 21JAN2014 ;

OUTPUT:

$10^{\wedge} 9$ pseudorandom numbers generated and summed in 46.213 seconds.

Their average is 3.999998 .

RandI() Probability Density Function and Simple Example

$$
f(k)=\left\{\begin{array}{c}
\frac{1}{b-a+1}, \text { for } a \leq k \leq b \\
0, \quad \text { otherwise }
\end{array}\right.
$$

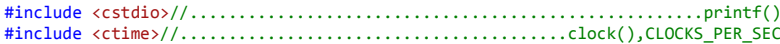

$\#$ include $" y \_r a n d o m . h " / / \ldots \ldots \ldots \ldots \ldots \ldots \ldots \ldots \ldots \ldots \ldots \ldots \ldots \ldots \ldots \ldots \ldots \ldots \ldots \ldots \ldots \ldots$
int $\operatorname{main}()\{/ /<================$ SIMPLE EXAMPLE FOR THE yRandom: $:$ RandI () FUNCTION

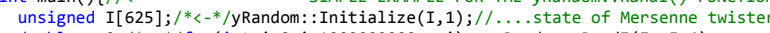
double $s=0 ; / *<-* /$ for (int $i=0 ; i<1000000000 ;++i) s+=y \operatorname{Random:~:~RandI~}(I,-5,4)$;

printf( $" 10^{\wedge} 9$ pseudorandom numbers generated and summed in $\% .3 f$ seconds. In

"Their average is \%f. \n\n", double(clock())/CLOCKS_PER_SEC, s/1000000000),

OUTPUT:

$10^{\wedge} 9$ pseudorandom numbers generated and summed in 10.140 seconds.

Their average is -0.500057 .

RandP() Probability Density Function and Simple Example

$$
f(k)=\frac{\mu^{k} e^{-\mu}}{k !}
$$

\#include <cstdio $>/ /$

.

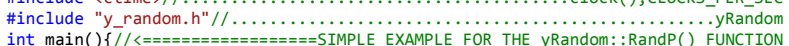
int unsigned I[625]; /*<-*/yRandom: : Initialize(I 1) ;/l yRato : Rand ( FUNCTION unsigned $\mathrm{I}=0 ; / *<-* /$ for (int $i=0 ; i<1000000000 ;++i) \mathrm{s}+=y$ Random: : RandP $(\mathrm{I}, 1)$; printf("10^9 pseudorandom numbers generated and summed in \%. $3 \mathrm{f}$ seconds. In" "Their average is \%f. In $\backslash n$ ", double(clock())/CLOCKS_PER_SEC, s/1000000000);

OUTPUT:

$10^{\wedge} 9$ pseudorandom numbers generated and summed in 35.708 seconds. 


\section{References}

1. Matsumoto, M.; Nishimura, T. Mersenne Twister: A 623-Dimensionally Equidistributed Uniform Pseudo-Random Number Generator. ACM Transactions on Modeling and Computer Simulation 1998, 8, (1), 3-20.

2. American National Standards Institute Standard INCITS/ISO/IEC 14882-2011. Information Technology - Programming Languages $-C++2012$.

3. Nishimura, T; Matsumoto, M. A C-Program for MT19937, With Initialization Improved, 26 January 2002. http://www.math.sci.hiroshima-u.ac.jp/ m-mat/MT/MT2002/CODES /mt19937ar.c. (accessed 12 December 2013).

4. Box, G. E.; Muller, M. E. A Note on the Generation of Random Normal Deviates. The Annals of Mathematical Statistics 1958, 29 (2), 610-611.

5. Abramowitz, M., Stegun, I. A., Eds. Handbook of Mathematical Functions With Formulas, Graphs, and Mathematical Tables; Applied Mathematics Series 55; U.S. National Bureau of Standards: Washington, DC, December 1972, section 7.1.26.

6. Knuth, D. E. Seminumerical Algorithms, the Art of Computer Programming; Vol. 2; Addison Wesley: Reading, MA, 1997. 


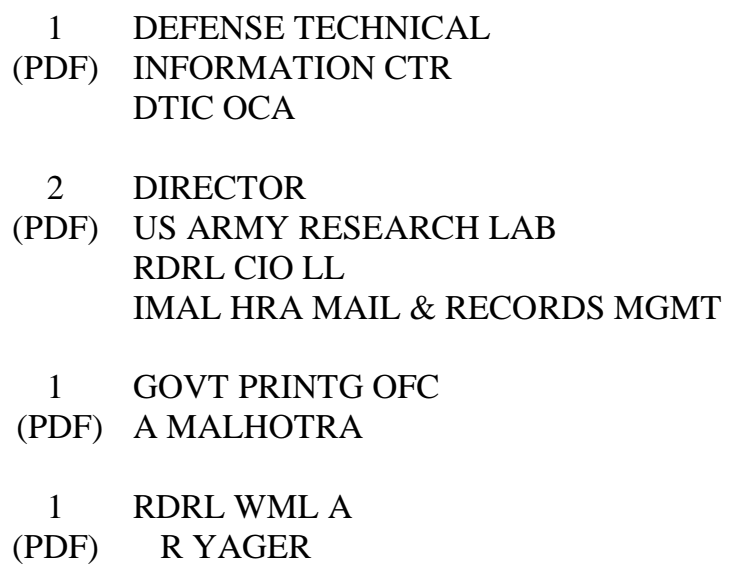


INTENTIONALLY LEFT BLANK. 